\title{
Author Correction: Viral programming of progenitor cell commitment
}

Matthew B. Reeves

Correction to: Nature Microbiology https://doi.org/10.1038/s41564-018-0136-4, published online 27 March 2018.

In the version of this News \& Views originally published, ref. 6 was incorrectly cited instead of ref. 5 at the end of the sentence shown below. This has now been corrected.

"Indeed, a proof of concept study has shown that latently expressed US28 can be targeted using an immunotoxin-based approach to eliminate infected cells in vitro 5 ."

Published online: 8 June 2018

https://doi.org/10.1038/s41564-018-0183-x 